\title{
Evaluation of CD147 gene expression, lipid peroxidation, and antioxidants in cases of acute coronary syndrome in Egyptian population
}

\author{
Wafaa A. Emam ${ }^{1}$, Nader M. M. Ali ${ }^{1}$, Aliaa T. A. Kamel ${ }^{1}$, Mohamed I. M. Eladawy ${ }^{2}$ and Nermin Raafat ${ }^{1 *}$ (D)
}

\begin{abstract}
Background: The main mechanism of acute coronary syndrome (ACS) is the rupture of atherosclerotic plaques. Matrix metalloproteinases (MMPs) play an important role in the rupture of the vulnerable plaques. MMP secretion is stimulated by CD147, one of the immunoglobulin families. Malondialdehyde is an important marker of oxidative damage, which is related to the atherosclerotic process. Superoxide dismutase normally prevents the oxidative process. This study was conducted to evaluate the association of ACS with CD147 gene expression, lipid peroxidation, and antioxidants in Egyptian population. The study included 124 people, 62 ACS patients and 62 healthy controls.
\end{abstract}

Results: CD147 gene expression in the ACS group was significantly increased compared to the control group $(p<$ 0.001). The ACS was $9.71 \pm 3.56$-fold; the control group was $0.94 \pm 0.19$-fold. Also, the SOD activity in the ACS group was significantly increased when compared to the control group ( $t=16.023, p<0.001)$. There was a highly significant increase in the MDA level in ACS groups when compared to the control group $(t=35.536, p<0.001)$. There was a highly significant increase in the creatine kinase-MB (CK-MB) and high sensitive troponin I levels in ACS groups when compared to the control group $(p<0.001)$.

Conclusion: There is a highly significant positive correlation between CK-MB and CD147 in both control and ACS groups ( $p=<0.001^{* *}$ ); also, there is highly significant positive correlation between high sensitive troponin I and CD 147 in both control and ACS groups $\left(p=<0.001^{* *}\right.$ ), but we did not find significant correlation between SOD and CD147 or between MDA and CD 147 in both control and ACS groups.

Keywords: CD147 gene, Lipid peroxidation, Antioxidants, Acute coronary syndrome

\section{Background}

Acute coronary syndrome (ACS) is a serious subtype of coronary heart disease (CHD); it is characterized by a progressive inflammation of the vascular wall and considered as a fundamental cause of death [1]. Acute coronary syndrome describes types of myocardial ischemic states that include unstable angina (UA), non-ST-elevated myocardial infarction (NSTEMI), and ST-elevated myocardial infarction (STEMI) [2].

\footnotetext{
* Correspondence: nerminraafat@gmail.com

${ }^{1}$ Medical Biochemistry Department, Faculty of Medicine, Zagazig University, Zagazig, Egypt

Full list of author information is available at the end of the article
}

Metalloproteinases (MMPs) are a family of latent zincand calcium-dependent enzymes, and they are responsible for the breakdown of the extracellular matrix in many diseases [3]. MMPs play a vital role in the rupture of the susceptible plaques by degrading the fibers of the plaque $[4,5]$, as they can be secreted from the activated vascular smooth muscle cells in the atherosclerotic plaques. Rupture of the atherosclerotic plaque is the main mechanism of ACS [6].

Extracellular MMP inducer (EMMPRIN) CD147 is responsible for the synthesis of these MMPs [7, 8]. CD147 or EMMPRIN is one of the immunoglobulin superfamily in humans, which plays an important role in the development 
of many cancers by stimulating the secretion of MMPs and cytokines. CD147 also regulates cell proliferation, apoptosis, tumor cell migration, metastasis, and differentiation, so it is considered as the main marker for inflammation $[9,10]$.

In addition, CD147 is important in processes of atherogenesis, atheroprogression, acute atherosclerothrombosis, and angiogenesis that play a critical role in advanced atherosclerotic plaques, which is believed to promote plaque destabilization [11]. All the previous factors may support many impaired pathways of atherosclerotic plaques leading to possible rupture of the vulnerable plaques [12].

Higher levels of CD 147 expression were detected in CAD patients. These high levels are not only detected in the plasma but also on platelets, monocytes, and granulocytes in the circulation [13].

The accumulation of free radicals has a crucial role in the development of atherosclerosis. Lipids especially low-density lipoproteins (LDL) are susceptible to be attacked by free radicals $[14,15]$. Oxidative modified LDL (Ox-LDL) has a vital role in the progression of the pathological state of atherosclerosis. Uptake of Ox-LDL by macrophages as well as smooth muscle cells causes the formation of foam cells, which is an important step in the development of atherosclerosis. Oxidative activities of the free radicals are revealed by measuring their oxidative yields in biological systems [16, 17]. Malondialdehyde (MDA) is the result of lipid peroxidation and its measurement is considered as an important marker of oxidative damage. So MDA is a useful marker for identification and further assessment of patients with CAD [18].

Some of the most imperative free radicals in biological systems are oxygen derivatives or reactive oxygen species (ROS). ROS molecules are characterized by the presence of one or more unpaired electrons plus their instability and high reactivity. They also interact rapidly with surrounding molecules, altering their structure and functions, so they are involved mainly in the pathogenesis of atherosclerosis and coronary heart diseases. Normally, these processes are largely nonpathogenic to the host organism because low levels of ROS are maintained by enzymatic scavenger mechanisms presented by antioxidant enzymes like catalase, glutathione peroxidase, and superoxide dismutase [19].

In this study, we aimed to evaluate the association of ACS with CD147 gene expression, lipid peroxidation, and antioxidants in Egyptian population.

\section{Methods}

The study was conducted in the Medical Biochemistry department. The study included 124 people, 62 ACS patients and 62 apparently healthy controls. This study has been approved by the Faculty of Medicine, Zagazig University, Institutional Review Board (IRB), for human studies (reference number is 2735/27-3-2016), and the patients have signed an informed written consent.

\section{Blood sampling}

Peripheral blood mononuclear cells (PBMCs) were purified from peripheral blood by standard density-gradient centrifugation using lymphocyte separation medium (Ficoll). In brief, blood was diluted 1:3 with sterile phosphate-buffered saline then became layered over the separation medium then centrifuged at $2000 \mathrm{rpm}$ for $20 \mathrm{~min}$ at $20^{\circ} \mathrm{C}$ temperature using a cooling centrifuge. PBMC layers were carefully aspirated after centrifugation.

\section{Real-time quantitative PCR analysis for expression of CD147 gene}

The total RNA was extracted from PBMCs following the manufacturer's instructions. Relative mRNA expression was performed by two-step qRT-PCR. RNA was reversetranscribed to synthesize cDNA using iNtRON Biotechnology kit. The amplification of specific RNA was performed in a $20-\mu \mathrm{l}$ reaction mixture containing $5 \mu \mathrm{l}$ of cDNA template, $10 \mu \mathrm{l}$ Eva Green mix (Jena Bioscience), and 100 $\mathrm{pmol} / \mu \mathrm{l} \mathrm{l}(0.5 \mu \mathrm{l})$ of each primer. The amplification was carried out using real-time PCR (StratageneMx3005PqPCR System). $\beta$-Actin was used as a housekeeping gene. Primer pair sequence for CD147 gene was CD147-F: CAG AGT GAA GGC TGT GAA GTCG; CD147-R: TGC GAG GAA CTC ACG AAG AA and for $\beta$-actin was $\beta$-actin-F: GGT TCC GCT GCC CTG AGG; $\beta$-actin-R: GTC CAC GTC ACA CTT CATG. qPCR reactions were as follows: initial denaturation and polymerase activation at $95^{\circ} \mathrm{C}$ for $2 \mathrm{~min}$, then 40 cycles of denaturation $95^{\circ} \mathrm{C}$ for $15 \mathrm{~s}$, annealing and elongation at $58^{\circ} \mathrm{C}$ for $1 \mathrm{~min}$. Relative changes in gene expression were calculated using the $2^{-\Delta \Delta \mathrm{CT}}$ method, described by Livak and Schmittgen [20].

\section{Measurement of MDA serum level}

Serum samples for measurement of MDA were obtained from venous blood by centrifugation of clotted specimen within $30 \mathrm{~min}$, and samples were kept frozen at $-70{ }^{\circ} \mathrm{C}$ until assays were carried out. Thiobarbituric acid (TBA) reacts with malondialdehyde (MDA) in acidic medium at a temperature of $95^{\circ} \mathrm{C}$ for $30 \mathrm{~min}$ to form a thiobarbituric acid reactive product with pink color, which is read at $534 \mathrm{~nm}$ [21].

\section{Measurement of SOD activity level}

This assay depends on the ability of SOD to inhibit phenazine methosulphate-mediated reduction of nitroblue tetrazolium dye. EDTA whole blood samples were washed 4 times with $0.9 \% \mathrm{NaCl}$ solution. The samples were centrifuged and the plasma was aspirated off. RBCs were washed 4 times with $3 \mathrm{ml} 0.9 \% \mathrm{NaCl}$ solution with centrifugation after each wash. The washed centrifuged RBCs were made up to $2 \mathrm{ml}$ with cold redistilled water, mixed and left to stand at $4{ }^{\circ} \mathrm{C}$ for $15 \mathrm{~min}$, then assayed for SOD according to the manufacturer's instructions of Abcam 
(Catalog no ab65354). If the samples were not assayed immediately, they were stored at $-70^{\circ} \mathrm{C}$ and then the lysate was diluted with distilled water. A spectrophotometer was used at a wave length of $450 \mathrm{~nm}$ [22].

\section{Statistical analysis}

Data analysis was performed using SPSS (statistical package for the social science) version 20. Data are summarized as the mean \pm standard deviation. To compare means, an independent sample $t$ test was used when appropriate. Nonparametric test (Mann-Whitney) was used to compare means when data was not normally distributed and to compare medians in categorical data. Correlations were performed using Pearson's correlation.

\section{Results}

There was a highly significant increase of CD147 gene expression, SOD activity, and MDA level in ACS groups when compared to the control group $(t=19.368, p<$ $0.001 ; t=16.023, p<0.001$; and $t=35.536, p<0.001$, respectively) as shown in Table 1.

Also, there was a highly significant increase in the CK$\mathrm{MB}$ and high sensitive troponin I levels in ACS groups when compared to the control group $(p<0.001)$ as shown in Table 2.

We found that there is no significant correlation between SOD and CD 147 gene expression in both control $(r=-0.016, p=0.902)$ and ACS groups $(r=$ $0.112, p=0.386$ ), and also, there is no significant correlation between MDA and CD147 gene expression in both control $(r=0.023, p=0.858)$ and ACS groups $(r=0.187, p=0.146)$ as shown in Table 3.

There is a highly significant positive correlation between CK-MB and CD147 gene expression in both control $(r=$ $0.976, p=<0.001)$ and ACS groups $(r=0.870, p=<0.001)$. There is also a highly significant positive correlation between high sensitive troponin I and CD147 gene expression

Table 1 A statistical comparison of CD147 gene expression, SOD activity, and MDA level among studied groups

\begin{tabular}{|c|c|c|c|c|}
\hline & ACS, $N=62$ & Controls, $N=62$ & $t$ test & $P$ \\
\hline \multicolumn{5}{|c|}{ CD147 gene fold change } \\
\hline Mean \pm SD & $9.71 \pm 3.56$ & $0.94 \pm 0.19$ & 19.368 & $<0.001^{* *}$ \\
\hline Range & $4.2-23.9$ & $0.65-1.78$ & & \\
\hline \multicolumn{5}{|l|}{ SOD } \\
\hline Mean \pm SD & $91.64 \pm 8.55$ & $73.8 \pm 1.93$ & 16.023 & $<0.001^{* *}$ \\
\hline Range & $70.22-115.62$ & $70.23-78.32$ & & \\
\hline \multicolumn{5}{|l|}{ MDA } \\
\hline Mean \pm SD & $3.82 \pm 0.24$ & $2.02 \pm 0.32$ & 35.536 & $<0.001^{* *}$ \\
\hline Range & $3.06-4.45$ & $1.53-2.6$ & & \\
\hline
\end{tabular}

${ }^{* *} p \leq 0.001$ is highly significant
Table 2 A statistical analysis of CK-MB and high sensitive troponin I levels in the studied groups

\begin{tabular}{lclll}
\hline & ACS, N=62 & Control, $N=62$ & MW test & $P$ \\
\hline CK-MB & & & & \\
Mean \pm SD & $59.59 \pm 26.23$ & $6.28 \pm 0.46$ & -9.596 & $<\mathbf{0 . 0 0 1 * *}$ \\
Range & $15.76-100.76$ & $1.77-19.55$ & & \\
High sensitive troponin I & & & \\
Mean \pm SD & $148.83 \pm 61.82$ & $0.94 \pm 0.5$ & -9.605 & $<0.001^{* *}$ \\
Range & $79.33-320.77$ & $0.15-2.01$ & & \\
\hline
\end{tabular}

MW Mann-Whitney test

${ }^{* *} p \leq 0.001$ is highly significant

in both control $(r=0.877, p=<0.001)$ and ACS groups $(r=0.850, p=<0.001)$ as shown in Table 4 .

\section{Discussion}

Acute coronary syndrome is considered as a severe subtype of coronary heart diseases. It is due to progressive inflammation of the vascular wall, and it is considered as the most common cause of mortality worldwide [1]. The key mechanism of ACS is the rupture of atherosclerotic plaques. MMPs play the main role in the rupture of the vulnerable plaques by degrading the fibrous tissue of the plaques $[4,5]$.

CD147, a member of the immunoglobulin superfamily, is a potent inducer of extracellular MMP, lymphocyte development, and immune response [23]. Higher levels of CD147 expression were detected in CAD patients. These high levels were not only detected in the plasma but also on platelets, monocytes, and granulocytes in the circulation. The severe situation of CAD is highly correlated with the level of CD147 on platelets [13]. The CD147 level was very high in ACS when compared with SA patients and the control group. Also, the higher levels of CD147 in the plasma were found in culprit vessels. These findings were strong evidence that CD147 was more relevant to unstable plaques and acute vascular diseases [12].

In our research, we found that the mean value of CD147 gene expression in the ACS group was significantly highly increased when compared to the control group. This finding could be explained on the basis of the identification of CD147 as a potent activator of MMP in adjacent fibroblasts through the homotypic CD147-CD147 interaction [24, 25]. Moreover, CD147

Table 3 Correlation of CD147 gene fold change with SOD and MDA

\begin{tabular}{|c|c|c|c|c|}
\hline & \multicolumn{2}{|c|}{$\mathrm{ACS}, N=62$} & \multicolumn{2}{|c|}{ Control, $N=62$} \\
\hline & $r$ & $p$ & $r$ & $p$ \\
\hline SOD & 0.112 & 0.386 & -0.016 & 0.902 \\
\hline MDA & 0.187 & 0.146 & 0.023 & 0.858 \\
\hline
\end{tabular}


Table 4 Correlation of CD147 gene fold change with CK-MB and high sensitive troponin I levels

\begin{tabular}{|c|c|c|c|c|}
\hline & \multicolumn{2}{|c|}{$\mathrm{ACS}, N=62$} & \multicolumn{2}{|c|}{ Control, $N=62$} \\
\hline & $r$ & $p$ & $r$ & $p$ \\
\hline CK-MB & 0.870 & $<0.001^{* *}$ & 0.976 & $<0.001^{* *}$ \\
\hline High sensitive troponin I & 0.850 & $<0.001^{* *}$ & 0.877 & $<0.001^{* *}$ \\
\hline
\end{tabular}

${ }^{* *} p \leq 0.001$ is highly significant

has recently been recognized as a potent marker of inflammation [26]. It has also a vital role in the complex process of atherogenesis, atheroprogression, acute atherosclerothrombosis, and angiogenesis, which play a critical role in advanced atherosclerotic plaques. Furthermore, it is believed to promote plaque destabilization [11]. All the previous factors may support many impaired pathways of atherosclerotic plaques leading to possible rupture of the vulnerable ones [12].

MMPs become activated by complex activation cascade: the pro-MMPs become activated by plasmin or by binding to membrane-anchored MMPs [27, 28]. So membrane type 1 MMP (MT1-MMP) facilitates all associated proteolysis and enhances monocyte migration and transmigration through activated endothelial cells [29]. MMP9 is highly found in the plasma of MI patients [30].

In a study by Schmit et al. [8], they discovered that in vitro EMMPRIN induce MMPs in SMCs (MMP-2) and monocytes (MMP-9). This assumes that the expression of EMMPRIN is greatly involved in MT1MMP expression and secretion of MMP9 in acute MI. They also approved that EMMPRIN-hindering will abrogate the activity of MMP-9. These findings suggest that EMMPRIN may not only induce MMPs directly but also play an important role as a key element in MMP induction.

In 2015, Sturhan et al. [31] proved that there is a significant difference in monocyte subtype distribution between healthy subjects and patients with stable CAD and no significant difference between acute MI patients and stable CAD one. This finding proves that the upregulation of EMMPRIN is associated with the rupture of the plaques by MMP-9 and followed by MI. EMMPRIN can function as an adhesion receptor. Platelets induce a pro-inflammatory phenotype in monocytes via CD147. The upregulation of CD147 gene expression in ACS affects monocyte differentiation and upregulates EMMPRIN in unstable patients as rupture plaques in acute MI are associated with a high expression level of CD147 gene. All these results go hand in hand with our findings.

SOD activity in this study showed a highly significant increase in the mean value in the diseased group when compared to the control one $(p<0.001)$. Increased SOD activity can predict a worse outcome in CAD patients [32]. In CAD, increased activity of SOD is not good, as it leads to peroxidation of lipids and hypersensitivity to oxidative stresses [33]. The main function of SOD is the conversion of superoxide anion to $\mathrm{H}_{2} \mathrm{O}_{2}$ [34] but increased SOD activity will shift the equilibrium equation, and this will lead to decreased $\mathrm{H}_{2} \mathrm{O}_{2}$ concentration [35].

Alongside with these findings, Vichova and Motovska in 2013 [36] reported that interruption of blood flow in the coronary arteries causes ischemia of the supplied tissues leading to their injury, necrosis, and apoptosis. During ischemia, cellular defense against oxidative injury is impaired with lower activities of SOD, glutathione peroxidase, and huge amount of ROS produced. A second explanation is that SOD is essential for radical disposal so that the low radical concentration is important to stop lipid peroxidation. If these chain of reactions are scavenged by decreased superoxide radical, this will lead to the whole reaction to be stopped or terminated [37]. A third explanation is that increasing SOD activity results in an increase of $\mathrm{H}_{2} \mathrm{O}_{2}$ concentration [38].

Brown and Griendling [39] reported that the presence of ROS is responsible for the increased activity of SOD as a result of inflammation and neutrophil migration. Also, Buettner et al. [33] suggested that SOD is not like GPX-1 as the increase in SOD activity has no protective effect but is associated with the deregulation of oxidative processes leading to severe tissue damage and an adverse outcome.

MDA is the result of lipid peroxidation. It is an undependable marker of oxidative damage, so the measurement of the MDA level is considered as a useful indicator and marker for the identification and assessment of ACS patients [18]. During myocardial ischemia or ongoing MI, high levels of free radicals may be generated. MDA that is generated from breakdown of lipids during peroxidation processes is considered as a reliable marker of oxidative damage [40].

Furthermore, Sharma et al. in 2008 [41] reported that the serum MDA level is increased in CAD patients significantly when compared to the control group. Also, Yaghoub et al. [42] proved that the serum level of MDA is considered as a potent marker of lipid peroxidation in CAD patients when compared to the control group, and this increase is correlated with the disease severity. In agreement with the previous authors, we found a significant increase in MDA in the ACS group when compared to the control one $(p<0.001)$.

High sensitive cardiac troponin I assay enables accurate quantification of troponin in most of healthy people. Moreover, these assays can help the assessment of patients with chest pain through the development of safe strategies to exclude myocardial infarction [43, 44]. Coven et al. [45] also demonstrated that the current definition of NSTEMI requires a typical clinical syndrome plus elevated troponin or CK-MB. They also found that cardiac-specific troponins are not detectable in the blood 
of healthy individuals. Therefore, they provide high specificity for detecting injury of cardiac myocytes. These molecules are also more sensitive than CK-MB for myocardial necrosis and consequently improve early detection of small myocardial infarctions.

Alvin et al. [46], on the contrary, found that CK-MBtesting provides no incremental value to patient care and its elimination can lead to millions of health care dollars saved without adversely affecting patient care. In our work, we found a highly significant positive correlation between the levels of CD147 gene expression, CK-MB and high sensitive troponin I in both studied groups.

These results agree completely with the findings reported by Sturhan et al. [31]. The explanation of our findings simply depends on the following facts: interaction of monocytes and platelets via CD147 leads to inflammation and differentiation [47]. There is no doubt that platelets induce pro-inflammatory phenotype in monocytes by CD147 as platelets play an important role in coronary heart disease, and this will speculate that the upregulation of CD147 in ACS must affect monocyte differentiation in their inflammatory activity [48]. The expression levels of healthy subjects were similar to patients with stable CAD. This means that the upregulation of CD147 on monocytes must be associated with unstable patients such as plaque rupture in acute MI [31].

Current care pathways are unable to exclude out MI presentation that is requiring admission to hospital in most patients, although most of them have no myocardial infarction and must be discharged from the hospital. High sensitive troponin I assay is a helpful and effective strategy to exclude MI [2].

\section{Conclusion}

We concluded that the positive correlation between high sensitive troponin I and CD147 gene expression will definitely diagnose MI. In summary, EMMPRIN may represent promising targets in atherosclerosis to prevent inflammatory activities leading to the progression of atherosclerosis and plaque rupture. In vivo studies are needed to evaluate the therapeutic potential of EMMPRIN in atherosclerosis.

\footnotetext{
Abbreviations

ACS: Acute coronary syndrome; CD147: Cluster of differentiation 147; CHD: Coronary heart disease; EMMPRIN: Extracellular matrix metalloproteinase inducer; LDL: Low-density lipoproteins; MDA: Malondialdehyde; MMPs: Matrix metalloproteinases; NSTEMI: Non-STelevated myocardial infarction; ROS: Reactive oxygen species; UA: Unstable angina
}

\section{Acknowledgements}

Not applicable

\section{Authors' contributions}

WAE prepared the idea and designed the study. NMMA did the data statistical analysis. ATAK and NR performed all the laboratory investigations and interpreted the patients' data regarding each studied group. MIME selected the patients and the control group. All authors wrote, read, and approved the final manuscript.

\section{Funding}

Not applicable

Availability of data and materials

Not applicable

Ethics approval and consent to participate

This study has been approved by the Faculty of Medicine, Zagazig University, Institutional Review Board (IRB), for human studies (reference number is 2735/27-3-2016) and the patients have signed an informed written consent.

Consent for publication

Not applicable

\section{Competing interests}

All authors declared that they have no competing interests.

\section{Author details}

${ }^{1}$ Medical Biochemistry Department, Faculty of Medicine, Zagazig University, Zagazig, Egypt. ${ }^{2}$ Cardiology Department, Faculty of Medicine, Zagazig University, Zagazig, Egypt.

Received: 14 October 2019 Accepted: 11 February 2020

Published online: 16 March 2020

\section{References}

1. Virmani R, Kolodgie FD, Burke AP, Farb A, Schwartz SM (2000) Lessons from sudden coronary death: A comprehensive morphological classification scheme for atherosclerotic lesions. Arteriosclerosis, thrombosis, and vascular biology 20(5):1262-1275 https://doi.org/10.1161/01.ATV.20.5.1262 PMID: 10807742

2. Thygesen K, Alpert JS, Jaffe AS, Simoons ML, Chaitman BR, White HD, Mendis S, the Joint ESC/ACCF/AHAMHF Task force for the universal definition of myocardial infarction (2012) Third universal definition of myocardial infarction. Circulation 126(16):2020-2035 https://doi.org/10.1161/ CIR.0b013e31826e1058 PMID:22923432

3. Woessner JF Jr (1991) Matrix metalloproteinases and their inhibitors in connective tissue remodeling. FASEB J 5(8):2145-2154 https://doi.org/10. 1096/fasebj.5.8.1850705 PMID:1850705

4. Lusis AJ (2000) Atherosclerosis. Nature 407(6801):233-241 https://doi.org/10. 1038/35025203 PMID:11001066

5. Libby $P$ (2001) Current concepts of the pathogenesis of the acute coronary syndromes. Circulation 104(3):365-372 https://doi.org/10.1161/01.CIR.104.3. 365 PMID:11457759

6. Galis ZS, Sukhova GK, Kranzhöfer R, Clark S, Libby P (1995) Macrophage foam cells from experimental atheroma constitutively produce matrixdegrading proteinases. Proc Natl Acad Sci U S A 92(2):402-406 https://doi. org/10.1073/pnas.92.2.402 PMID:7831299

7. Biswas C, Zhang Y, DeCastro R, Guo H, Nakamura T, Kataoka H, Nabeshima K (1995) The human tumor cell-derived collagenase stimulatory factor (renamed EMMPRIN) is a member of the immunoglobulin superfamily. Cancer Res 55(2):434-439 PMID:7812975

8. Schmidt R, Bültmann A, Ungerer M, Joghetaei N, Bülbül $\mathrm{O}$, Thieme $\mathrm{S}$ et al (2006a) Extracellular matrix metalloproteinase inducer regulates matrix metalloproteinase activity in cardiovascular cells: implications in acute myocardial infarction. Circulation 113(6):834-841 https://doi.org/10.1161/ CIRCULATIONAHA.105.568162 PMID:16461815

9. Gwinn WM, Damsker JM, Falahati R, Okwumabua I, Kelly-Welch A, Keegan AD et al (2006) Novel approach to inhibit asthma-mediated lung inflammation using anti-CD147 intervention. J Immunol 177(7):4870-4879 https://doi.org/10.4049/jimmunol.177.7.4870 PMID:16982929

10. Zhang J, Ge H, Wang C, Guo T, He Q, Shao Q et al (2007) Inhibitory effect of PPAR on the expression of EMMPRIN in macrophages and foam cells. Int J Cardiol 117(3):373-380 https://doi.org/10.1016/j.ijcard.2006.05.023

11. Joghetaei N, Stein A, Byrne RA, Schulz C, King L, May AE et al (2013) The Extracellular Matrix Metalloproteinase Inducer (EMMPRIN, CD147) - a potential novel target in atherothrombosis prevention? Thrombosis Res 
131(6):474-480 https://doi.org/10.1016/j.thromres.2013.04.017 PMID: 23669100

12. Yan J, Mao Y, Wang C, Wang Z (2015) Association study between an SNP in CD147 and its expression with acute coronary syndrome in a Jiangsu Chinese population. Medicine 94(42):e1537 https://doi.org/10.1097/MD. 0000000000001537 PMID:26496256

13. Pennings GJ, Yong AS, Kritharides L (2010) Expression of EMMPRIN (CD147) on circulating platelets in vivo. J Thromb Haemost 8(3):472-481 https://doi. org/10.1111/j.1538-7836.2009.03716.x PMID:19995409

14. Kannel WB (1998) Overview of atherosclerosis. Clin Ther 20(20):B2-B17 https://doi.org/10.1016/S0149-2918(98)80027-1)

15. Lloyd-Jones DM, Larson MG, Beiser A, Levy D (1999) Lifetime risk of developing coronary heart disease. Lancet 353(9147):89-92 https://doi.org/ 10.1016/S0140-6736(98)10279-9 PMID:10023892

16. Köse K, Doğan P (1995) Lipoperoxidation induced by hydrogen peroxide in human erythrocyte membranes. 2. Comparison of the antioxidant effect of Ginkgo biloba extract (EGb 761) with those of water-soluble and lipidsoluble antioxidants. J Int Med Res 23(1):9-18 https://doi.org/10.1177/ 030006059502300102 PMID:7774762

17. Miyazaki T, Shimada K, Sato O, Kotani K, Kume A, Sumiyoshi K et al (2005) Circulating malondialdehyde-modified LDL and atherogenic lipoprotein profiles measured by nuclear magnetic resonance spectroscopy in patients with coronary artery disease. Atherosclerosis 179(1):139-145 https://doi.org/ 10.1016/j.atherosclerosis.2004.09.013 PMID:15721020

18. Khaki-Khatibi F, Yaghoubi A, Rahbani N (2013) Study of antioxidant enzymes, lipid peroxidation, lipid profile and immunologic factor in coronary artery disease in East Azarbijan. Int J Med Biomed Res 1(2):147-152 https://doi.org/10.14194/ijmbr.1210

19. Cooke MS, Evans MD, Dizdaroglu M, Lunec J (2003) Oxidative DNA damage: mechanisms, mutation, and disease. FASEB J 17(10):1195-1214 https://doi. org/10.1096/fj.02-0752rev PMID:12832285

20. Livak KJ, Schmittgen TD (2001) Analysis of relative gene expression data using real-time quantitative PCR and the $2(-\Delta \Delta C(T))$ method. Methods 25(4):402-408 https://doi.org/10.1006/meth.2001.1262 PMID:11846609

21. Satoh K (1978) Serum lipid peroxide in cerebrovascular disorders determined by a new colorimetric method. Clinica Chimica Acta 90(1):3743 https://doi.org/10.1016/0009-8981(78)90081-5 PMID:719890

22. Nishikimi M, Roa NA, Yogi K (1972) The occurrence of supeoxide anion in the reaction of reduced phenazine methosulfate and molecular oxygen. Biochem Biophys Res Commun 46:849-854. https://doi.org/10.1016/S0006291X(72)80218-3

23. lacono KT, Brown AL, Greene MI, Saouaf SJ (2007) CD147 immunoglobulin superfamily receptor function and role in pathology. Exp Mol Pathol 83(3): 283-295 https://doi.org/10.1016/j.yexmp.2007.08.014 PMID:17945211

24. Suzuki S, Sato M, Senoo H, Ishikawa K (2004) Direct cell-cell interaction enhances pro-MMP-2 production and activation in co-culture of laryngeal cancer cells and fibroblasts: involvement of EMMPRIN and MT1-MMP. Exp Cell Res 293(2):259-266 https://doi.org/10.1016/j.yexcr.2003.10.010 PMID: 14729463

25. Yan L, Zucker S, Toole BP (2005) Roles of the multifunctional glycoprotein, emmprin (basigin; CD147), in tumour progression. Thromb Haemo 93(2): 199-204 PMID:15711733

26. Galis ZS, Khatri JJ (2002) Matrix metalloproteinases in vascular remodeling and atherogenesis: the good, the bad, and the ugly. Circ Res 90(3):251-262

27. Seizer P, Geisler T, Bigalke B, Schneider M, Klingel K, Kandolf R et al (2013) EMMPRIN and its ligand cyclophilin A as novel diagnostic markers in inflammatory cardiomyopathy. Int J Cardiol 163(3):299-304 https://doi.org/ 10.1016/j.jijcard.2011.06.049 PMID:21724278

28. Rajavashisth TB, Xu XP, Jovinge S, Meisel S, Xu XO, Chai NN et al (1999) Membrane type 1 matrix metalloproteinase expression in human atherosclerotic plaques: evidence for activation by proinflammatory mediators. Circulation 99(24):3103-3109 https://doi.org/10.1161/01.CIR.99.24. 3103 PMID:10377072

29. Matías-Román S, Gálvez BG, Genís L, Yáñez-Mó M, de la Rosa G, SánchezMateos P et al (2005) Membrane type 1-matrix metalloproteinase is involved in migration of human monocytes and is regulated through their interaction with fibronectin or endothelium. Blood 105(10):3956-3964 https://doi.org/10.1182/blood-2004-06-2382 PMID:15665118

30. Kai H, Ikeda H, Yasukawa H, Kai M, Seki Y, Kuwahara F et al (1998) Peripheral blood levels of matrix metalloproteases-2 and -9 are elevated in patients with acute coronary syndromes. J Am Coll Cardiol 32(2):368-372 https://doi. org/10.1016/S0735-1097(98)00250-2 PMID:9708462

31. Sturhan $H$, Ungern-Sternberg $S N$, Langer $H$, Gawaz M, Geisler T, May AE, Seizer P (2015) Regulation of EMMPRIN (CD147) on monocyte subsets in patients with symptomatic coronary artery disease. Thromb Res 135(6): 1160-1164 https://doi.org/10.1016/j.thromres.2015.03.022 PMID:25824988

32. Zengin E, Sinning C, Zeller T, Rupprecht HJ, Schnabel RB, Lackner KJ et al (2015) Activity of superoxide dismutase copper/zinc type and prognosis in a cohort of patients with coronary artery disease. Biomarkers Med 9(6):597604 https://doi.org/10.2217/bmm.15.23 PMID:26079963

33. Buettner GR (2011) Superoxide dismutase in redox biology: the roles of superoxide and hydrogen peroxide. Anticancer Agents Med Chem 11(4): 341-346. https://doi.org/10.2174/187152011795677544

34. Fridovich I (1995) Superoxide radical and superoxide dismutases. Annu Rev Biochem 64(1):97-112 https://doi.org/10.1146/annurev.bi.64.070195.000525 PMID:7574505

35. Kowald A, Klipp E (2004) Alternative pathways might mediate toxicity of high concentrations of superoxide dismutase. Ann N Y Acad Sci 1019(1): 370-374 https://doi.org/10.1196/annals.1297.065 PMID:15247047

36. Vichova T, Motovska Z (2013) Oxidative stress: predictive marker for coronary artery disease. Exp Clin Cardiol 18(2):e88-e91

37. Mccord J, M. (1993) Human disease, free radicals, and the oxidant/ antioxidant balance. Clin Biocheist 26(5):351-357 https://doi.org/10.1016/ 0009-9120(93)90111-I

38. Kowald A, Lehrach H, Klipp E (2006) Alternative pathways as mechanism for the negative effects associated with overexpression of superoxide dismutase. J Theor Biol 238(4):828-840 https://doi.org/10.1016/j.jtbi.2005.06.034

39. Brown DI, Griendling KK (2015) Regulation of signal transduction by reactive oxygen species in the cardiovascular system. Circ Res 116(3):531-549 https://doi.org/10.1161/CIRCRESAHA.116.303584 PMID:25634975

40. Pucheu S, Coudray C, Vanzetto G, Favier A, Machecourt J, de Leiris J (1995) Assessment of radical activity during the acute phase of myocardial infarction following fibrinolysis: utility of assaying plasma malondialdehyde. Free Rad Biol Med 19(6):873-881 https://doi.org/10.1016/08915849(95)94361-G PMID:8582662

41. Sharma SB, Garg S, Veerwal A, Dwivedi S (2008) hs-CRP and oxidative stress in young CAD patients: a pilot study. Ind J Clin Biochem 23(4):334-336 https://doi.org/10.1007/s12291-008-0073-8 PMID:23105781

42. Yaghoubi A, Ghojazadeh M, Abolhasani S, Alikhah H, Khaki-Khatibi F (2015) Correlation of serum levels of vitronectin, malondialdehyde and Hs- CRP with disease severity in coronary artery disease. J Cardiovasc Thorac Res 7(4):113-117

43. Apple FS, Ler R, Murakami MM (2012) Determination of 19 cardiac troponin I and T assay 99th percentile values from a common presumably healthy population. Clin Chem 58(11):1574-1581 https://doi.org/10.1373/clinchem. 2012.192716 PMID:22983113

44. Carlton E, Greenslade J, Cullen L, Body R, Than M, Pickering JW et al (2016) Evaluation of high-sensitivity cardiac troponin I levels in patients with suspected acute coronary syndrome. JAMA Cardiol 1(4):405-412 https://doi. org/10.1001/jamacardio.2016.1309 PMID:27438316

45. Alvin MD, Jaffe AS, Ziegelstein RC, Trost JC (2017) Eliminating creatine kinase-myocardial band testing in suspected acute coronary syndrome: a value-based quality improvement. JAMA Int Med 177(10):1508-1512 https:// doi.org/10.1001/jamainternmed.2017.3597 PMID:28806444

46. Covn DL, Kalyanasundaram A, Shirani J (2018) Acute coronary syndrome workup. Emedicine Medecape last updated on Sept 05, 2018

47. Wrigley BJ, Shantsila E, Tapp LD, Lip GY (2013) Increased formation of monocyte-platelet aggregates in ischemic heart failure. Circulation 6(1):127135 https://doi.org/10.1161/CIRCHEARTFAILURE.112.968073 PMID:23152489

48. Rong MY, Wang CH, Wu ZB, Zeng W, Zheng ZH, Han Q et al (2014) Platelets induce a proinflammatory phenotype in monocytes via the CD147 pathway in rheumatoid arthritis. Arth Res Ther 16(6):478 https://doi.org/10.1186/ s13075-014-0478-0 PMID:25404518

\section{Publisher's Note}

Springer Nature remains neutral with regard to jurisdictional claims in published maps and institutional affiliations. 